\title{
Dynamic posture analysis of Spacelab-1 crew members
}

\author{
D. J. Anderson ${ }^{1}$, M.F. Reschke ${ }^{2}$, J.E. Homick ${ }^{2}$, and S. A. S. Werness ${ }^{3}$ \\ ${ }^{1}$ Department of Electrical Engineering and Computer Science and the Kresge Hearing Research Institute, \\ The University of Michigan, Ann Arbor, MI 48109, USA \\ ${ }^{2}$ Space Biomedical Research Institute, NASA-Johnson Space Center, Houston, TX 77058, USA \\ ${ }^{3}$ Environmental Research Institute of Michigan, Ann Arbor, MI 48105, USA
}

Summary. Dynamic posture testing was conducted on the science crew of the Spacelab-1 mission on a single axis linear motion platform. Tests took place in pre- and post-flight sessions lasting approximately 20 min each. The pre-flight tests were widely spaced over the several months prior to the mission while the post-flight tests were conducted over the first, second, fourth, and sixth days after landing. Two of the crew members were also tested on the day of landing. Consistent with previous postural testing conducted on flight crews, these crew members were able to complete simple postural tasks to an acceptable level even in the first few hours after landing. Our tests were designed to induce dynamic postural responses using a variety of stimuli and from these responses, evaluate subtle changes in the postural control system which had occurred over the duration of the flight. Periodic sampling post-flight allowed us to observe the time course of readaptation to terrestrial life. Our observations of hip and shoulder position, when subjected to careful analysis, indicated modification of the postural response from pre- to postflight and that demonstrable adjustments in the dynamic control of their postural systems were taking place in the first few days after flight. For transient stimuli where the platform on which they were asked to stand quickly moved a few centimeters fore or aft then stopped, ballistic or open loop 'programs' would closely characterize the response. During these responses the desired target position was not always achieved and of equal importance not always properly corrected some 15 seconds after the platform ceased to move. The persistent observation was that the subjects had a much stronger dependence on visual stabilization post-flight than pre-flight. This was best illustrated by a slow or only partial recovery to an upward posture after a transient base-of-

Offprint requests to: A.J. Anderson (address see above) support movement with eyes open. Postural responses to persistent wideband pseudorandom base-of-support translation stimuli were modeled as time invarient linear systems arrived at by Kalman adaptive filter techniques. Derived model parameters such as damping factor and fundamental frequency of the closed loop system showed significant modification between pre- and post-flight. This phenomenon is best characterized by movement of the poles toward increasing stability. While pre-flight data tended to show shoulders and hips moving in phase with each other, post-flight data showed a more disjoint behavior. One can speculate that this change illustrates a shattered postural organization or an acquired strategy not designed to stabilize terrestrial posture but as a carry over from optimum inflight postural control. Given our observations one can never be certain if these changes represent modifications in the physiology of posture of purposeful changes in strategy. As in other examples of motion behavior, the time domain analysis as represented by the step changes in position is not always reconcilable with the system modeling of pseudorandum responses and subsequent frequency domain analysis as represented by the pseudorandom noise stimuli. We present the observed data with arguments and some contradictions as to the nature of the adaptive changes which occur in the postural control system.

Key words: Posture - Adaptation - Space flight

\section{Introduction}

Modifications to posture have long been recognized as an adaptation to new motion environments and 
indeed new modes of transportation. As in the case of passengers and crews of ocean going vessels, this adaptation in space craft crews is often accompanied by nausea and frank illness resulting in loss of valuable work time from duties. While maintenance of posture at sea or on any other earth bound vehicle in a complication of the fundamental problem of keeping the center of gravity over the base of support in the face of conflicting sensory information, space flight presents the additional complexity of, in effect, exchanging the necessity to stabilize the centers of gravity over the base of support for the necessity to engage in a completely different mode of locomotion.

Attempts have been made from the first extended orbital flights to document and quantify the exact nature of the postural adaptations (Graybiel and Fregly 1966; Homick and Miller 1975). While tests of balance using rails of varying widths were applied through Apollo to Skylab, there was increasing awareness that static balance tests could not uncover subtle changes in the subjects's ability to solve the dynamic posture problem presented by gait or movement of base of support (Homick 1977). Further, the emphasis was on the role of the vestibular system and although considerable knowledge was gained, the importance of the relative contributions of vision, vestibular input and proprioception were not revealed in detail.

Approaches to develop methods for posture analysis have followed two paths. One seeks to neutralize the input of one or the other of the sensory modalities (Nashner 1976; Nashner and Berthoz 1978). The other presents the subject with a number of base of support movements which challenge the postural system in different ways (Andres 1980) and utilizes system analysis techniques to infer mechanisms from the complex body movements which result. Assumptions on the structural complexity of the upright human form have been assumed starting from an inverted pendulum model (Nashner 1972) to a multilink model (Koozekanani et al. 1980). One notable characteristic of the latter method was the requirement for a vast amount of joint position data to solve the dynamics.

The following study utilizes a number of base of support movements which are familiar to the subjects and which occur quite naturally in everyday movement. The analysis spans from the presentation of the time series observations of transient hip and shoulder motion and EMG data in raw graphical form to the presentation of responses to pseudorandom motion of the base of support as derived results in the complex plane of the $\mathrm{Z}$ operator.

\section{Methods}

The dynamic posture platform used in these tests was developed for various testing conditions ranging from normal subjects to elderly clinical cases. Briefly, the level platform is driven fore and aft in a plane parallel to the floor by a direct current motor powered by a switching type servo amplifier. A digital computer commands the velocity servo in such a way as to produce a number of standard motions. The data recorded by the computer as the platform moved were platform position, platform velocity, subject shoulder and hip positions and two channels of EMG. The hip and shoulder measurements were made by two line scan cameras positioned to view the subject profile at the levels of interest as motion is induced by the platform. The use of our posture platform and the various types of analysis used on the data have been described elsewhere by Andres and Anderson (1980) and by Werness and Anderson (1984a, b).

The motion protocols conducted on the crew of Spacelab-1 were optimized to provide economy of total testing time. This was necessary because of the number of concurrent tests, the fatiguing nature of the tests and desirability of maintaining a high level of attention over the testing period. Before each session and sometimes in conjunction with the needs of other investigators, EMG electrodes were applied to record activity of the Soleus and Tibialis Anterior muscles. To provide consistent and accurate measurements from the line scan cameras, each subject was attired in clothing which was dark enough to provide a contrast with the background and be consistently detected by the measurement cameras. The clothing was also close fitting engough so that no relative motion occurred between body and clothing. After a brief platform calibration, the subject took a stance on the platform facing along the axis of platform motion and viewing a eye-level target on a wall approximately $4 \mathrm{ft}$ in front of him. The instructions were to stand comfortably with arms crossed and knees straight but not locked. The objectives of the instructions were to produce consistent behavior over the series and to limit motion of the knee joints thus providing a three link biomechanical model of the body.

No particular instructions were given as to platform tracking strategy but each subject had enough experience with the platform prior the test series being reported to establish his own strategy. As the each test session progressed, the subject was told to stand either with eyes closed (EC) or with eyes open (EO) but was not told which motion type would be coming next or when it would begin. Figure 1a illustrates the position of the subject and the motion direction of the platform.

There were four motion profile types used in our testing with a total of nine data trials: Two static trials with EC, one sinusoidal trial with EC, four transient trials with all combinations of forward (FW), backward (BW), EO and EC, and two pseudorandom trials one $\mathrm{EO}$ and one EC. A platform velocity sequence was precalculated for each trial and passed to the servo command loop by a Digital Equipment Corporation LSI/11 digital computer through digital to analog converters. Trials were approximately $30 \mathrm{~s}$ apart with the recorded portion lasting $20 \mathrm{~s}$. The characteristics of the four trial types is as follows:

\section{Static}

No motion of the platform occurred for the entire $20 \mathrm{~s}$ of analysis time.

\section{Sinusoidal}

The platform moved with a smooth sine-wave motion of $5 \mathrm{~s}$ per cycle with an excursion of about $14 \mathrm{~cm}$ (Fig. 1b). 


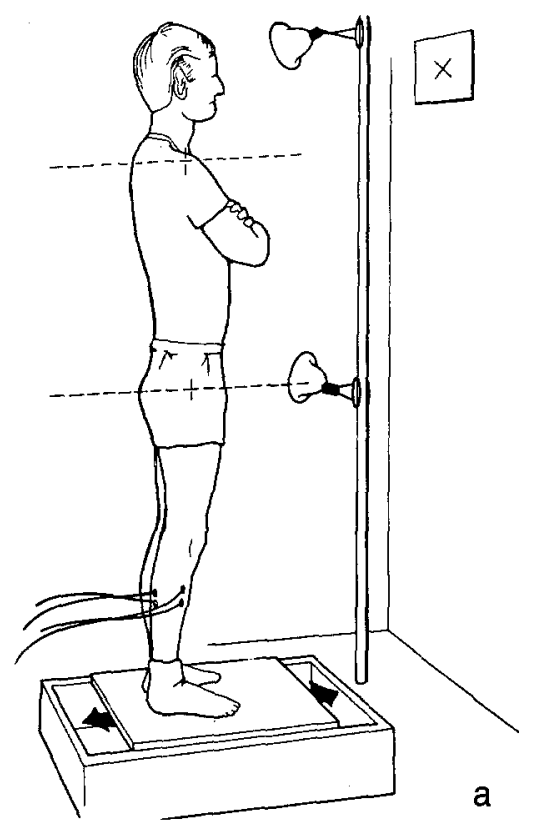

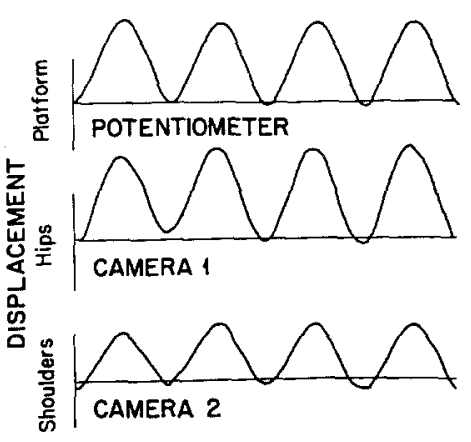
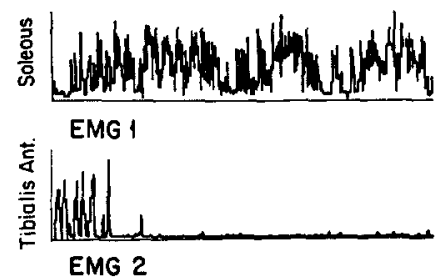

EMG 2
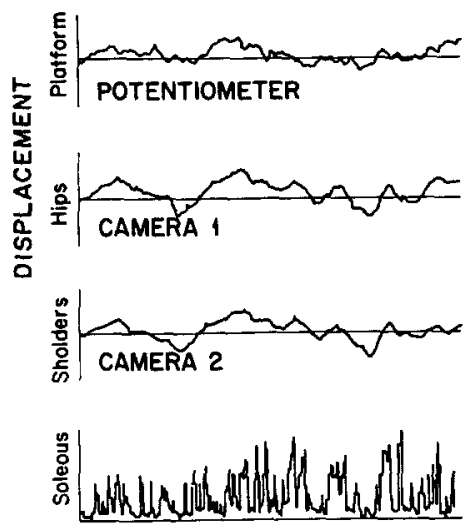

EMG 1

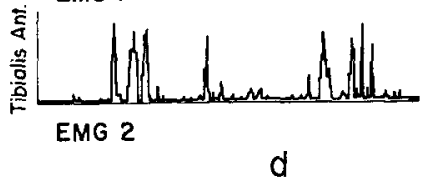

Fig. 1. The experimental configuration is shown (a) with the subject ready for a base of support motion stimulation. Backlighting is used to create a silhouette which can be detected by the line scan cameras. Panels b, $c$ and d illustrate typical data sets consisting of three position records and two EMG records obtained from the sinusoidal, transient and pseudorandom stimulus types
C

\section{Transient}

The platform moves FW or BW following a cosine velocity bell having a peak value of $7 \mathrm{~cm}$ and lasting $2 \mathrm{~s}$ (Fig. 1b). The stimulus starts without warning. Data are taken starting $5 \mathrm{~s}$ before the motion and continue through the motion for $20 \mathrm{~s}$ (Fig. 1c).

\section{Pseudorandom}

Velocity commands to the platform consisted of a random time series which had been bandlimited to $2 \mathrm{~Hz}$ through the use of an eliptical digital filter. The magnitude distribution was normalized to be between $7 \mathrm{~cm} / \mathrm{s}$ and $-7 \mathrm{~cm} / \mathrm{s}$ (Fig. 1d). For all tests occurring on day zero, this magnitude distribution was narrowed.

Data were collected in digital form using the same digital computer as was used to generate the stimuli. A record of each subject session was stored in a single file on a flexible disk which contained protocol information, platform calibration data and data from the nine subject trials. The data set format was common to all trials. Measured platform position and velocity were sampled at 25 $\mathrm{Hz}$ from the platform electronics. Shoulder and hip position data sampled at $25 \mathrm{~Hz}$ were obtained from digital linescan cameras viewing the subject from the side. Rectified and detected Soleus and Tibialis Anterior EMG data were sampled at $100 \mathrm{~Hz}$. The approximate levels of the line scan cameras are shown in Fig. 1a. Typical records obtained from each of the three active motion types are shown in Fig. 1b-c.

Transient responses were analyzed using primarily time domain techniques. Although one could define numerous parameters for quantification of these trials, the three measures chosen for illustrating the results of these tests were latency to EMG peaks, final error of the subject's shoulders to the platform and the speed of movement of the shoulders just after the platform ceased to move. Latency is measured by finding the highest value of a EMG burst and recording its time position from the beginning of the 

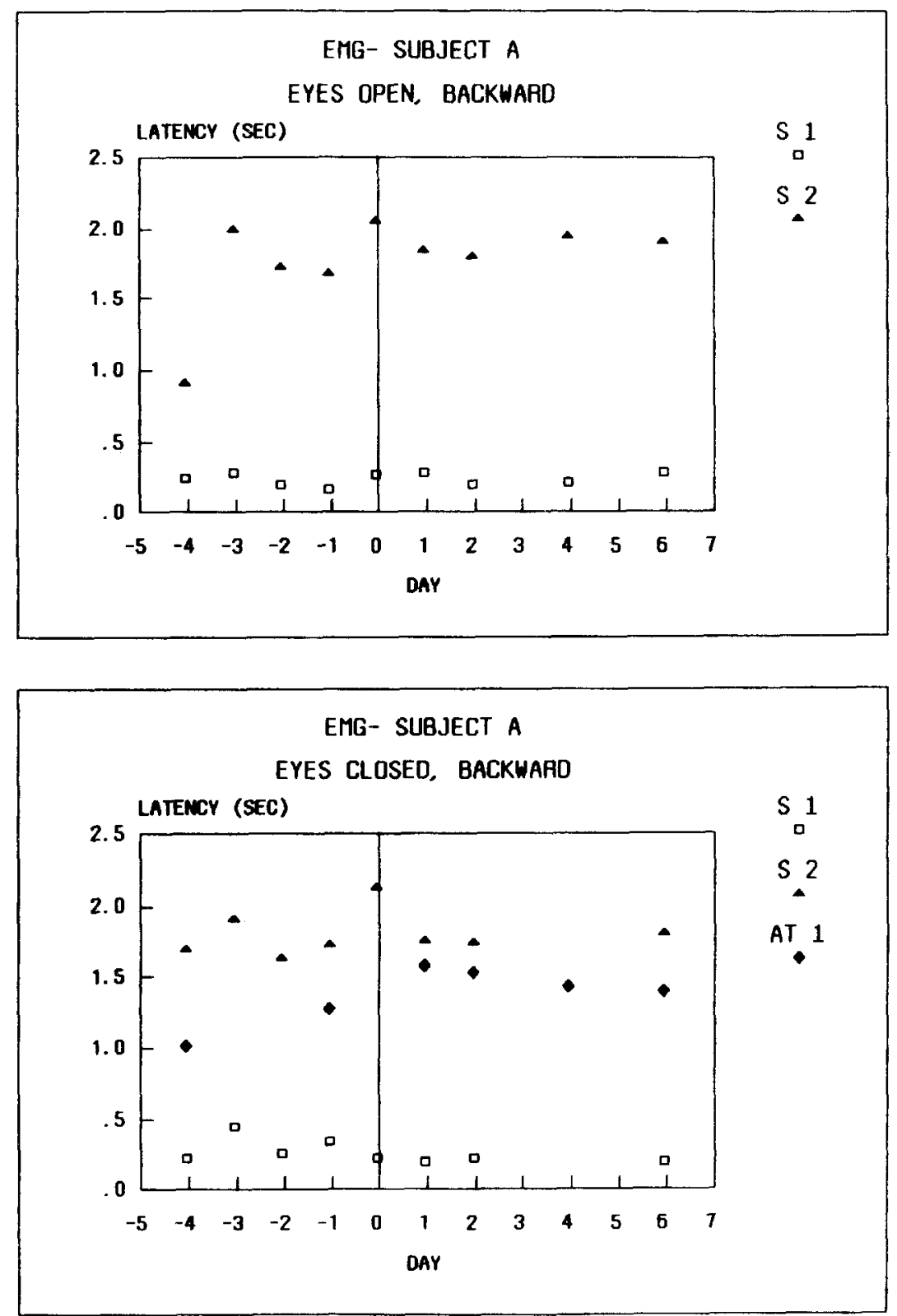

Fig. 2. EMG timing for transient stimuli tended to be consistent over the entire preand post-flight for both eyes open (top) and eyes closed (bottom). Three responses are recognized in the analysis. $\mathrm{S} 1$ - first soleus, $\mathrm{S} 2$ - second soleus and AT - anterior tibialis. All illustrations indicating days pre- and postflight are spaced over several months preflight but represent a true timefor post-flight data transient stimulation. Final error is defined for this paper as the difference between subject shoulder position and platform $15 \mathrm{~s}$ after the transient stimulation. Velocity of shoulder motion is measured just as the platform motion stops by a finite impulse response digital differentiator.

Most of the Pseudorandom stimulus analysis is carried out under the assumption that the postural control is a linear time invarient closed loop control system operating on the platform position as a single deterministic input. Since the data are in digital form within the computer, we use a discrete-time model which was both feedback and feedforward weighting factors. In addition, we have found it necessary to model delay and noise terms.

$$
y_{k}=\sum_{l=1}^{L} \alpha_{i} y_{k-1}+\sum_{m=0}^{M} b_{m-m} x_{k-m-d}+\varepsilon_{k}
$$

where $\varepsilon_{\mathrm{k}}$ is colored noise obtained from a white noise signal as follows:

$$
\varepsilon_{k}=\sum_{p=1}^{P} f_{p} \varepsilon_{k-p}+\underset{q=1}{Q} c_{q} e_{k-q}+e_{k}
$$

or in transform notation:

$$
Y(z)=\frac{z^{-d} B(z)}{A(z)} X(z)+\frac{C(z)}{F(z) A(z)} E(z)
$$

The complex root positions of polynomials $A(z)$ and $B(z)$ with respect to the unit circle in the plane of the complex variable $\mathrm{z}$ give important information on the damping factors and characteristic frequencies of the deterministic portion of the underlying control system model. Closeness to the unit circle indicates low damping while angle measured from the positive real axis indicates frequency. Since the sample rate for our data was $25 \mathrm{~Hz}$, rotation to the negative real axis indicates $12 \mathrm{~Hz}$. Poles or the roots of $\mathrm{A}(\mathrm{z})$ inside the unit circle indicate a stable control system. It is with 

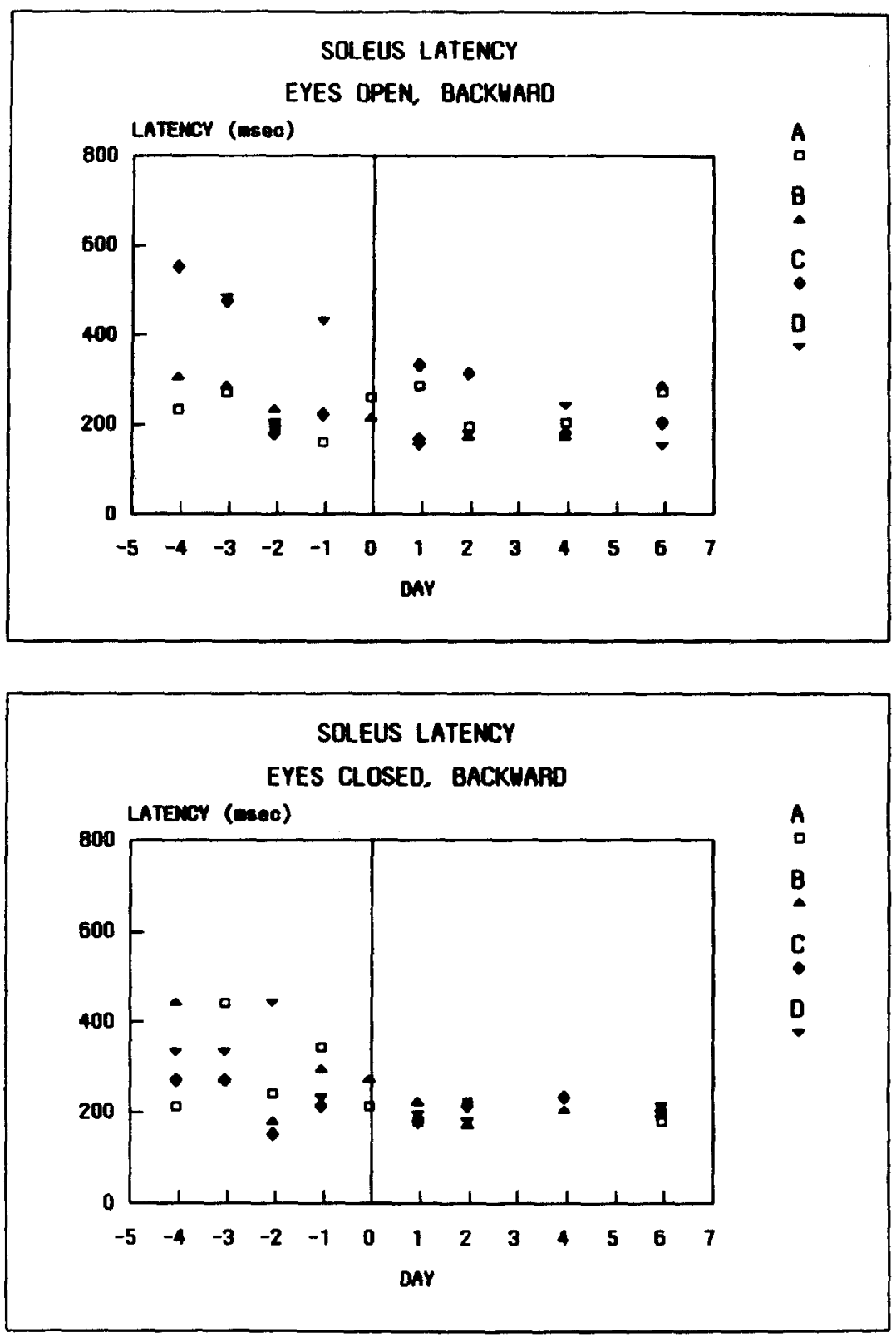

Fig. 3. When backward transients were applied, the consistency of the first Soleus muscle response between subjects and within subjects tends to increase. This is particularly evident in the eyes closed case but is also true for eyes open trials these radius and frequency parameters that explain our observations of responses to pseudorandom stimulation. More detail on the use of these models and the methods used to derive them are given by Werness et al. (1984a, b).

\section{Results}

The nine stimulus protocol delivered to each subject on each of the test days proved to be an efficient and fast way to acquire data on all stimuli within our experience without introducing undue fatigue or other complications to the testing protocols being used by other experimenters. We were limited to one sample of each test condition at each subject session. Below, the results from each stimulus condition will be presented.

\section{Statics and sinusoids}

The subjects did not show significant differences either from one another or pre- and post-flight through the static and sinusoid tests. It should be noted however that two of the crew volunteered subjective sensations of exaggerated fore and aft movements with the eyes closed under both experimental conditions. The sensations seemed to be 

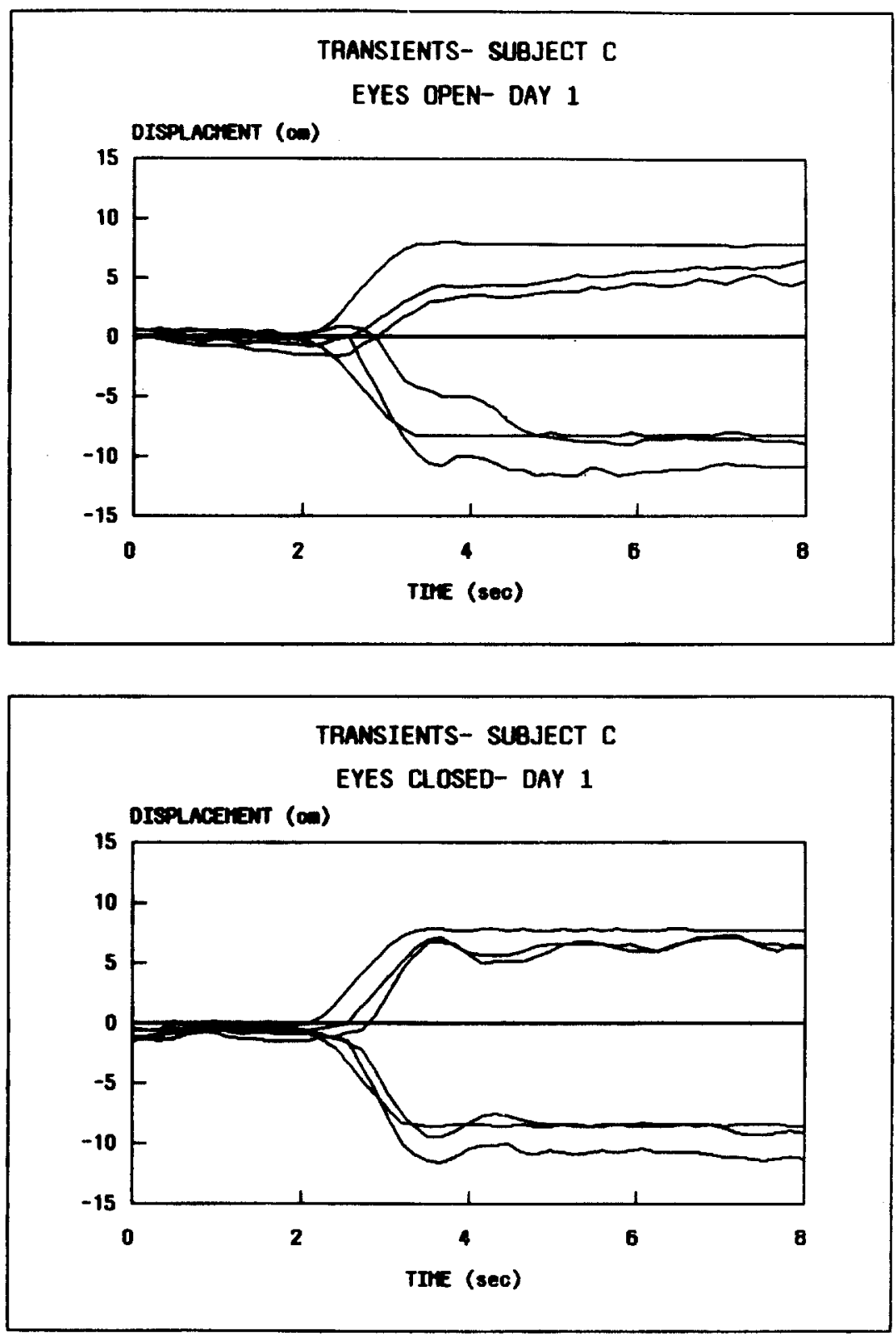

Fig. 4. While eyes are closed hip and shoulder responses post-flight tended to be consistent with each other, eyes open responses tended to stress the primary objective of visual stabilization related to the confusion of translational motion and postural sway. After static tests or when the platform was at rest for other reasons often a remark was ventured indicating a sensation of slow fore and aft motion of the platform. Surrounding the time of these remarks, various motions had been completed by the system but the reference was always to the static state of the platform.

\section{Transients}

Over a variety of normal and clinical subjects, we have found response to transient stimuli to be classified into only a few variations on the open loop or ballistic trajectory outlined by Bizzi et al. (1976) for volitional movements. That is, there were reciprocal bursts of EMG activity which placed the subject close if not on the target position for his center of gravity over his base of support. This two to four phase series of muscular events in the first hundred milliseconds was then followed by a more purposeful correction of a perceived error to the final error position. Since the biomechanical requirement for static postural stability is limited only to the requirement that the center of gravity is over any portion of the base of support, subjects can accept a wide range of initial and/or final positions. As a result, even for 

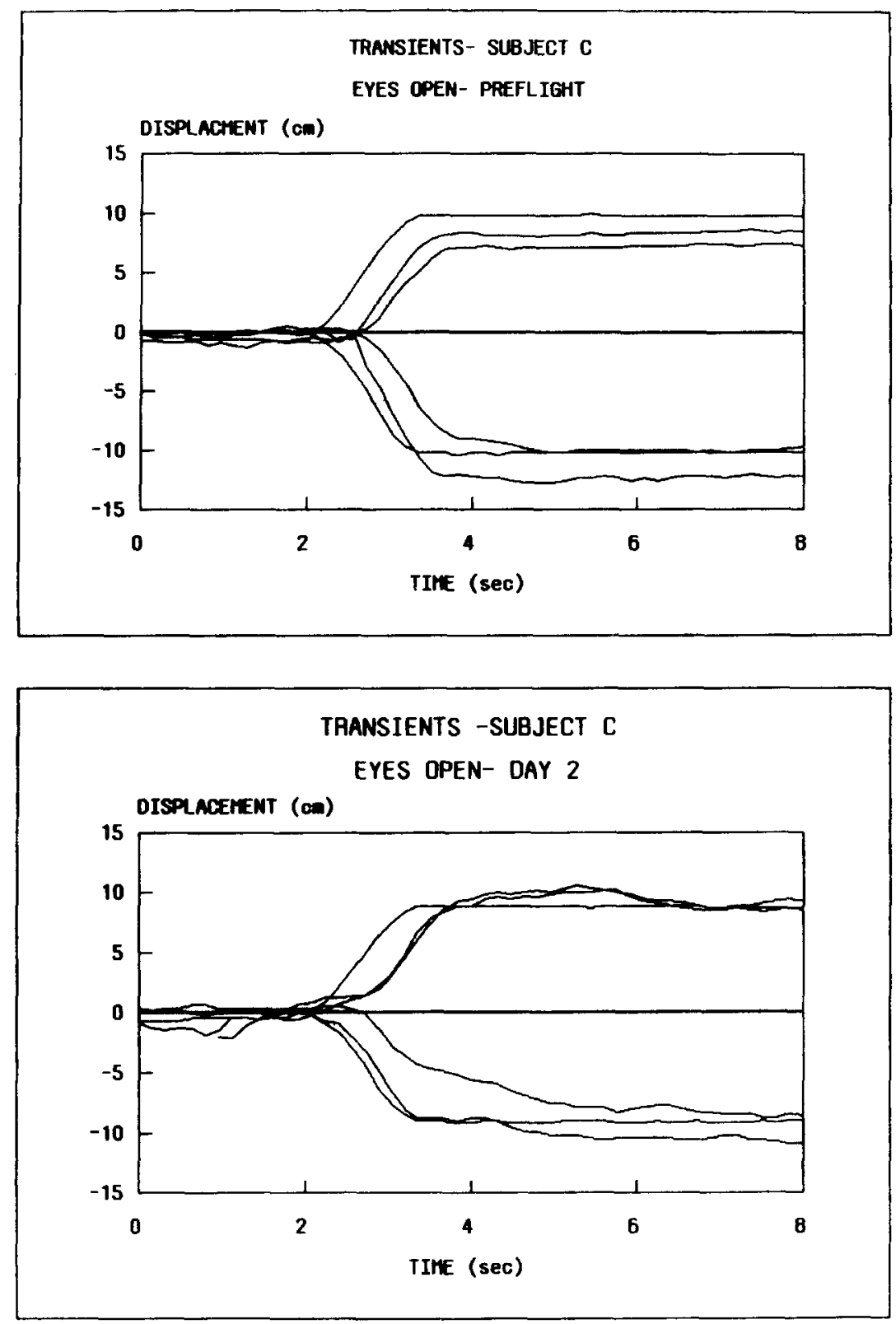

Fig. 5. In the top panel, a typical post-flight response is given with the day 2 response to the same stimulus. Each, by their slow return to alignment with the platform show some emphasis on visual stabilization for the shoulders but none of the oscillatory behavior seen on day 1 the same subject tested on successive days under nearly identical conditions the consistency of response is not always present. In fact, the most confident and least threatened subject sometimes has large errors between his initial and final position relative to the platform and as a consequence a large variation from trial to trial. Paradoxically, only the most unsure subjects exhibit a truly consistent pre and final position response consisting of almost perfect tracking of the platform with starting and final positions being within a few millimeters of each other.

For most clinical situations there is insufficient time to achieve total familiarity with the tests being conducted and therefore one might expect the results of successive trials to be effected by development of a strategy toward the equipment and the testing paradigm. The testing program for spacelab-1 provided considerable time for the subjects to form a strategy toward the platform and become consistent in their response to the different motions before the mission. One cannot be certain, however, that there was no strategy change between the testing session before flight and those after landing

Generally, the transient responses did not show the consistent rigid tracking response to the transient motion we expect from a subject or patient who is unsure of his stability. Instead, they showed the same 

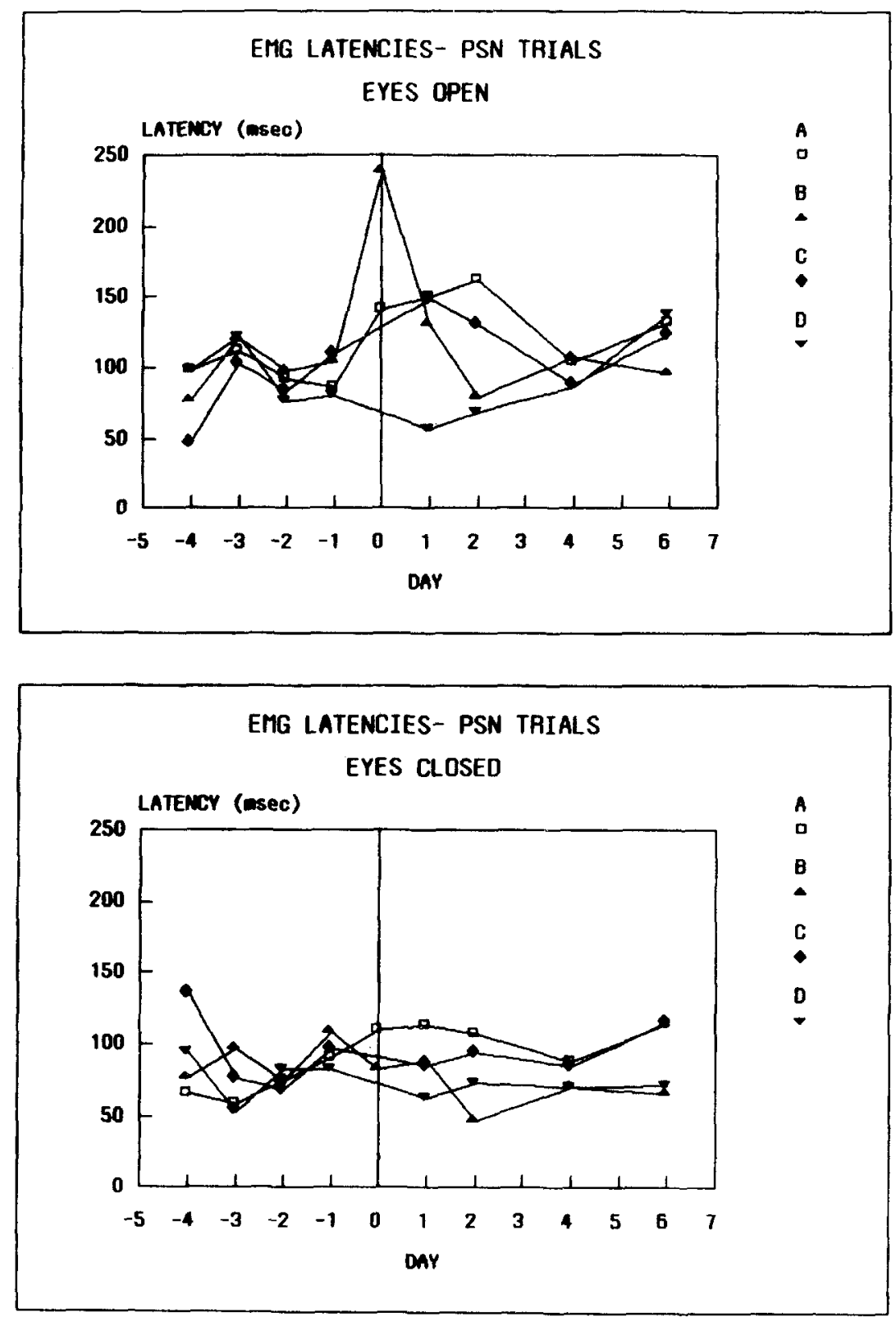

Fig. 6. EMG latencies derived from pseudorandom trials by correlating platform velocity with the Soleus EMG signal sown a lag early in the post-flight period for all but one of the test subjects. Subject D shows generally less adaptation activity during and after the flight variable response expected from a confident normal subject. In some cases there was a tolerance of final error which may indicate a lack of gravity perception. Shoulder final error for all subjects indicate that for eyes closed trials there is a strong tendency not to return to the full upright state. Examination of the position data for subjects $A$ and $B$ indicates this tolerance on days $+1,+2$ and +4 which was inconsistent with their past performance. Subjects C and D also showed some tolerance for error post-flight but also showed the same indifference pre-flight.

The EMG latency data for these subjects indicate no profound changes in overall response structure in that the same type of reciprocal muscle activation waves occur to achieve stability after a transient excitation for both pre- and post-flight data. Figure 2 illustrates this point for subject $\mathrm{A}$. The eyes open data indicate an early (S1) and a late (S1) soleus discharge but no tibialis anterior response. Eyes closed responses add a tibialis anterior (AT) discharge to the sequences. The subject has virtually the same participation and latency of response for each component throughout the entire schedule of postural testing pre- and post-flight.

When the latency data are examined on a finer scale and for all subjects as in Fig. 3, there is a strong suggestion that the consistency of Soleus muscle latency is greater post-flight than observed pre-flight 

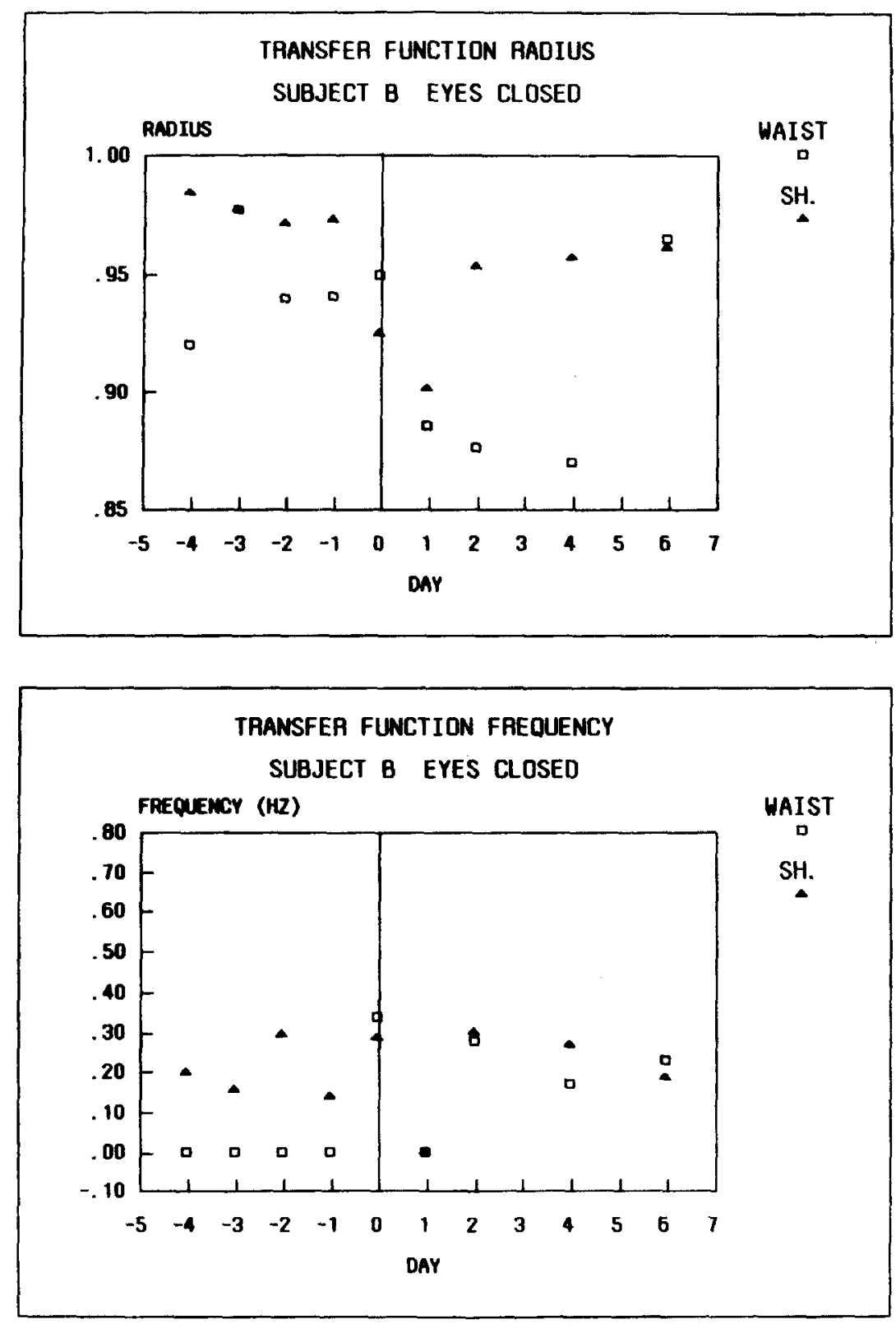

Fig. 7. Linear time invarient parametric models were derived from responses to pseudorandom stimuli. Pole positions of these models in the Z-plane indicate more stable hip movements post-flight for subject $B$. There is also change from real (zero frequency) to complex poles post-flight

for both eyes open and eyes closed conditions but more so for eyes closed.

While the muscle latency data show the same general pattern pre- and post-flight, the position data show dramatic intratrial changes. Day 1 data from subject C (Fig. 4) show two distinctive characteristics not shown in the pre-flight data. Both FW (shown as positive traces) and BW (shown as negative traces) movements under eyes open conditions indicate that the shoulders are slow to position themselves. In paticular, the BW movement is halting and even lags the waist movement meaning that there was considerable hip joint flexion as final posture was achieved. The EC conditions showed both strong tracking between shoulders and hips and a movement toward a more oscillitory behavior. Figure 5 indicates that the shoulder lag condition exists for EO BW on preflight days but is exaggerated post-flight. EO FW responses do not show the effect presumably because of the limits of hip flexion.

\section{Pseudorandom}

The response of a subject to pseudorandom stimuli may be conditioned as is his transient response but conditioning represents a setting of parameters which determine the dynamics of the postural system for a 

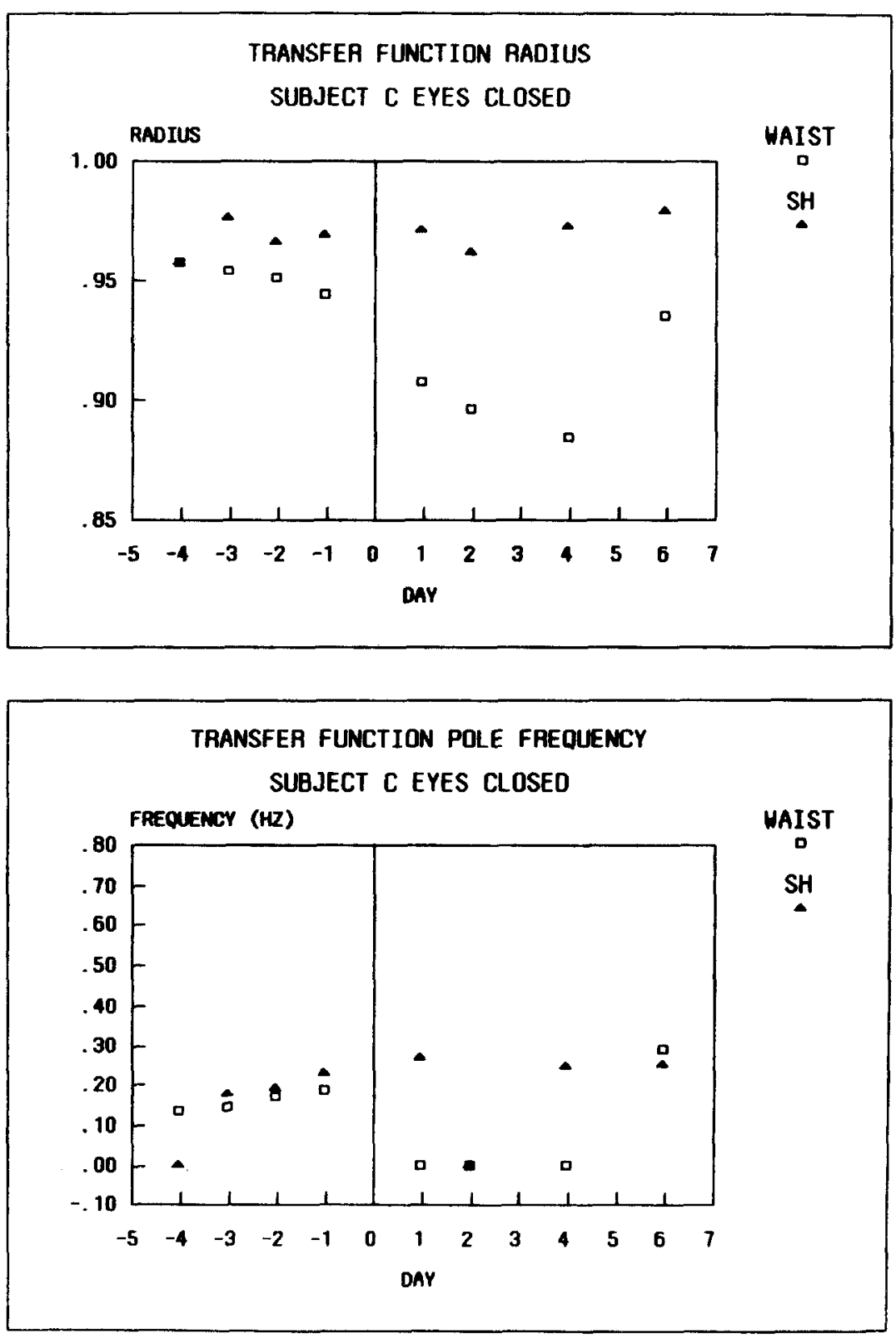

Fig. 8. Subject $C$ demonstrated similar changes in Z-plane pole radius as subject B (Fig. 7) but waist poles tended to go from complex to real post-flight. Both subjects had pole positions near pre-flight on the last testing day. More data for a longer period could have confirmed return to baseline for these parameters sustained 'weathering' of the stimulus rather than a strategy for limited action over a short period of time. Our analysis of both the EMG and the position data is therefore directed toward discovering these underlying parameters. Systems analysis techniques generally depend upon the ability to claim linearity of the system under analysis. Although this may be true to some degree for the position parameters we measure, the EMG responses do not have this property. For the above reason, the EMG data were analyzed using the cross-correlation statistic only. Shoulder and hip position data analysis, however, hypothesized adherence to a linear systems model as a characteristic of the system.
As shown in Fig. 1d, there is persistent EMG activity on the Soleus throughout a pseudorandom stimulation. Although this response may have a complex nonlinear relationship with the stimulus, the cross-correlation function still has meaning with respect to latency of response. Because of their lack of persistence, the Anterior Tibialis responses were not subjected to this analysis. Figure 6 shows the time shift in milliseconds to the peak of crosscorrelation functions between platform velocity and EMG magnitude calculated for all crew members for each pseudorandom response in the data set. With the exception of one crew member (D), the tendency was for longer latencies at the first three post-flight days 
with the EO conditions showing the greatest effects. The actual values of delay tend to be in the $100 \mathrm{~ms}$ range rather in the $200 \mathrm{~ms}$ range as would be predicted from the transient data.

It was determined by systematic analysis of all shoulder and hip data from the four crew members that there were no fundamental changes in model order for either the deterministic portion of the response or to the stochastic portion. When the model parameters were compared across subjects and pre- and post-flight days, the most remarkable changes were significant translation of transfer function pole positions in the complex $\mathrm{Z}$ plane. These changes were evident both in the radial direction (damping characteristic) and in the tangential direction (frequency). Subjects B (Fig. 7) and C (Fig. 8) are used for illustration below while subject $\mathrm{A}$ was slightly ambiguous and subject D showed little or no changes. Subject D's lack of posture modification pre- to post-flight in this test was consistent with his responses in other tests. For all subjects, EC data tended to be the most affected by the intervening space flight.

Figure 7 illustrates translations of B's transfer function poles between pre- and post-flight from an overdamped waist response (indicated by zero frequency in the lower panel) to an oscillatory but still heavily damped waist (low radius value). It should be noted that, during the period of observation, there may not be a return to baseline for this subject.

Damping characteristics of subject C's waist motions (Fig. 8) change from nearly undamped preflight (radius near unity) to highly damped post-flight as indicated by both smaller radius and zero frequency.

Both subject's models have increases in distance from the unit circle (shorter radius) for their postflight eyes closed waist response which indicates a movement toward greater stability. While there are no other significant changes in damping, both subjects showed changes in character of response from nonoscillatory to oscillatory or vice versa between pre- and post-flight sessions.

\section{Discussion}

Our data on responses to motions of base of support in subjects recovering from extended periods of weightlessness indicate a number of changes in postural control which are interesting in the context of our knowledge of posture and motion awareness. We tend to agree with the general principles outlined by Clément et al. (1984) who indicate that changes in postural control mechanisms tend to be conservative in the long term with perturbations to accommodate immediate situations. Changes we observed in all but one of our subjects were adjustments in postural control parameters but were not fundamental in nature. It should be noted that the subject (D) who had the most modest change of postural control had minimum difficulty adapting to microgravity and the least change in the in-flight experiments from day 2 to day 6.

It is not surprising that the subjects should have a much greater dependence on vision since they had been primarily dependent on that modality over the period of the flight. Static tilt has no meaning in flight and ankle joint torque is not experienced in flight in the context of postural stability. The degree of this dependence is evident from the slow recovery of shoulder position to alignment with the base of support after a transient motion.

While the EO responses tend to move toward greater stability under transient conditions, overshoot and oscillation tend to increase post-flight with EC indicating perhaps a movement toward tighter control or simply a more rigid ankle joint. We associate this with a decreased awareness of postural tilt as reported by the otolithic organs and interpreted centrally. Final error data which indicate acceptance of a large error in alignment with base of support after a transient motion tend to encourage this view.

Pseudorandom responses with EC tend to contradict the transient data because of the movement of system poles to greater stability post-flight verses greater oscillatory behavior under transient conditions. One possible explanation for this may be the persistence of the pseudorandom stimulus forcing the awareness of reduced stability early in the trials and cause a strategic change toward increased stability within the session. Our analysis procedures were not designed to detect such changes. The EMG latency data as developed from the pseudorandom trials, however, tend to support the EO results showing a slower response postflight for three of the four subjects.

Some of our data tended to indicate a greater independence of hip and shoulder post-flight. This, of course, indicates a departure from the inverted pendulum model of posture which is so often assumed. There may be three reasons for this behavior. The first is that the use of an extra joint in the control of posture can increase stability simply by adding resources to the catalog of motions. Two, it may be forced by the tendency of the shoulders to maintain their positions in space by forcing the hips to counter belance the center of mass. Three, one of the principal methods of controlling position in 
weightlessness is by modifications of the moment of inertia which can most easily be accomplished by bending a joint having masses attached on either side of it. The post-flight behavior may be a carry over from that environment.

None of the above changes in testing performance manifested themselves in gross differences in preand post-flight behavior and their effects seemed to disappear within the 6-day testing period. It would have been more helpful to observe the subjects over a longer period just to confirm that most parameters returned to baseline over the full testing period.

Acknowledgements. Support for this work was supplied by NASA through contracts NAS 9-15375 and NAS 9-15244. Over and above the financial support, many dedicated workers at several NASA centers worked long hours contributing their skills to make this experiment a success.

\section{References}

Anderson DJ, Homick JL, Jones KW (1977) Line scan cameras applied to posturography. In: Proceedings of the 1977 San Diego Biomedical Symposium, San Diego, pp 35-40

Andres RO, Anderson DJ (1980) Designing a better postural measurement system. Am J Otol 1: 197-206

Angel RW (1981) Electromyographic patterns during ballistic movements in normals and in hemiplegic patients. In:
Desmedt JE (ed) Progress in clinical neurophysiology, Vol 9. Motor unit types, recruitment and plasticity in health and disease. Jarger, Basel, pp 347-357

Bizzi E, Polit A, Morasso P (1976) Mechanisms underlying achievement of final head position. J Neurophysiol 39: 435-444

Clément G, Gurfinkel VS, Lestienne F, Lipshits MI, Popov KE (1984) Adaptation of postural control to weightlessness. Exp Brain Res 57: 61-72

Graybiel A, Fregly AR (1966) A new quantitative ataxia test battery. Acta Otolaryngol 69: 292-312

Homick JL, Miller EF (1975) Apollo flight crew vestibular assessment. In: Biomedical Results of Apollo. NASA SP-368

Homick JL, Reschke MF, Miller EF (1977) The effects of prolonged exposure to weightlessness on postural equilibrium. In: Biomedical Results From Skylab. NASA SP-377

Koozekanani SH, Stockwell CW, McGhee RB, Firoozmand M (1980) On the role of dynamic models in quantitative posturography. IEEE Trans Biomed Eng BME 27: 605-609

Nashner LM (1972) Vestibular posture control model. Kybernetik 10: $106-110$

Nashner LM (1976) Adapting reflexes controlling the human posture. Exp Brain Res 26: 59-72

Nashner LM, Berthoz A (1978) Visual contribution to rapid motor responses during posture control. Brain Res 150: 403-407

Werness SAS, Anderson DJ (1984a) A computer program for linear non-parametric and parametric identification of biological data. Comp Prog Biomed 18: 77-93

Werness SAS, Anderson DJ (1984b) Parametric analysis of dynamic postural responses. Biol Cybern 51: 155-168

Received September 13, 1985 / Accepted July 1, 1986 